
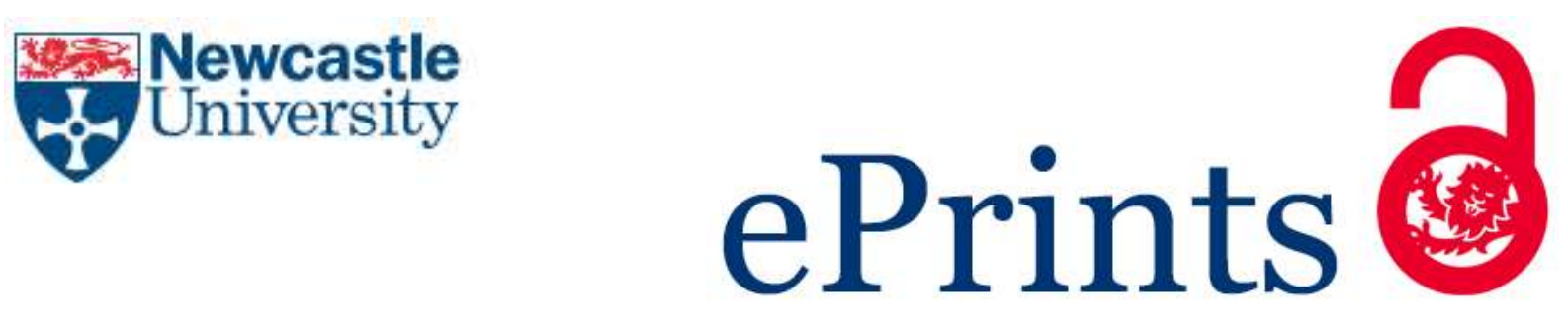

Lillethorup TP, Glud AN, Alstrup AKO, Mikkelsen TW, Nielsen EH, Zaer H, Doudet DJ, Brooks DJ, Sørensen JCH, Orlowski D, Landau AM.

Nigrostriatal proteasome inhibition impairs dopamine neurotransmission and motor function in minipigs.

Experimental Neurology 2018, 303, 142-152.

\title{
Copyright:
}

(C) 2018. This manuscript version is made available under the CC-BY-NC-ND 4.0 license

DOI link to article:

https://doi.org/10.1016/j.expneurol.2018.02.005

Date deposited:

$06 / 02 / 2018$

Embargo release date:

08 February 2019

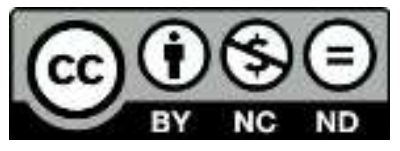

This work is licensed under a

Creative Commons Attribution-NonCommercial-NoDerivatives 4.0 International licence 


\section{Nigrostriatal proteasome inhibition impairs dopamine neurotransmission and motor function in minipigs}

Thea P. Lillethorup ${ }^{\mathrm{a}}$, Andreas N. Glud ${ }^{\mathrm{b}}$, Aage K. O. Alstrup ${ }^{\mathrm{a}}$, Trine W. Mikkelsen ${ }^{\mathrm{b}}$, Erik H. Nielsenª, Hamed Zaer $^{\mathrm{b}}$, Doris J. Doudet ${ }^{\mathrm{c}}$, David J. Brooks ${ }^{\mathrm{a}, \mathrm{d}, \mathrm{e}}$, Jens Christian H. Sørensen ${ }^{\mathrm{b}}$, Dariusz Orlowski ${ }^{\mathrm{b}} \&$ Anne M. Landau a, ${ }^{\text {a,* }}$

${ }^{a}$ Department of Nuclear Medicine and PET Center, Institute of Clinical Medicine, Aarhus University and Hospital, Denmark

${ }^{\mathrm{b}}$ Center for Experimental Neuroscience (Cense), Department of Neurosurgery, Institute of Clinical Medicine, Aarhus University, Denmark

${ }^{\mathrm{c}}$ Department of Medicine/Neurology, University of British Columbia, Vancouver, BC, Canada

${ }^{\mathrm{d}}$ Division of Neuroscience, Department of Medicine, Imperial College London, UK

${ }^{\mathrm{e}}$ Division of Neuroscience, Newcastle University, UK

${ }^{\mathrm{f}}$ Translational Neuropsychiatry Unit, Institute of Clinical Medicine, Aarhus University, Denmark

*Correspondence and requests for materials should be addressed to Anne M. Landau (alandau@clin.au.dk), Department of Nuclear Medicine and PET Center, Aarhus University, Nørrebrogade 44, Building 10G, $6^{\text {th }}$ floor, Aarhus C, Denmark 


\section{Abstract}

Parkinson's disease (PD) is characterized by degeneration of dopaminergic neurons in the substantia nigra leading to slowness and stiffness of limb movement with rest tremor. Using ubiquitin proteasome system inhibitors, rodent models have shown nigrostriatal degeneration and motor impairment. We translated this model to the Göttingen minipig by administering lactacystin into the medial forebrain bundle (MFB). Minipigs underwent positron emission tomography (PET) imaging with $(+)-\alpha-\left[{ }^{11} \mathrm{C}\right]$ dihydrotetrabenazine $\left(\left[{ }^{11} \mathrm{C}\right] \mathrm{DTBZ}\right)$, a marker of vesicular monoamine transporter 2 availability, at baseline and three weeks after the unilateral administration of $100 \mu \mathrm{g}$ lactacystin into the MFB. Compared to their baseline values, minipigs injected with lactacystin showed on average a $36 \%$ decrease in ipsilateral striatal binding potential corresponding to impaired presynaptic dopamine terminals. Behaviorally, minipigs displayed asymmetrical motor disability with spontaneous rotations in one of the animals. Immunoreactivity for tyrosine hydroxylase (TH) and HLA-DR-positive microglia confirmed asymmetrical reduction in nigral THpositive neurons with an inflammatory response in the lactacystin-injected minipigs. In conclusion, direct injection of lactacystin into the MFB of minipigs provides a model of PD with reduced dopamine neurotransmission, $\mathrm{TH}$-positive neuron reduction, microglial activation and behavioural deficits. This large animal model could be useful in studies of symptomatic and neuroprotective therapies with translatability to human PD.

\section{Keywords:}

Parkinson's disease, Proteasome inhibition, Lactacystin, PET imaging, Minipig, Vesicular monoamine transporter 2, Microglia

\section{Abbreviations:}

$\left[{ }^{11} \mathrm{C}\right] \mathrm{DTBZ}=(+)-\alpha-\left[{ }^{11} \mathrm{C}\right]$ dihydrotetrabenazine; $\alpha$-syn $=$ Alpha-synuclein; $B P_{N D}=$ non-displaceable binding potential; DVR $=$ Distribution volume ratio; Lac = Lactacystin; $\mathrm{MFB}=$ Medial forebrain bundle; $\mathrm{MHC}=$ Major histocompatibility complex; MPTP = 1-methyl-4-phenyl-1,2,3,6tetrahydropyridine; MRI = magnetic resonance image; $\mathrm{PD}=$ Parkinson's disease; $\mathrm{PET}=$ Positron emission tomography; $\mathrm{SN}=$ Substantia nigra; $\mathrm{SNc}=$ Substantia nigra pars compacta; $\mathrm{SNr}=$ Substantia nigra pars reticulata; $\mathrm{TH}$ = Tyrosine hydroxylase; UPS = Ubiquitin proteasome system; VMAT2 = Vesicular monoamine transporter 2; VTA $=$ Ventral tegmental area. 


\section{Introduction}

The ubiquitin proteasome system (UPS) is, together with the autophagy-lysosomal pathway, responsible for clearance of damaged intracellular proteins (Cuervo et al., 2004; Ebrahimi-Fakhari et al., 2011). Dysfunction of the UPS disturbs protein homeostasis which can lead to accumulation and aggregation of unwanted proteins as has been observed in Parkinson's disease (PD) where Lewy body inclusions and neurites containing aggregated alpha-synuclein ( $\alpha$-syn) are characteristically present (Betarbet et al., 2005; McNaught and Jenner, 2001). Lewy body inclusions in surviving neurons contain poly-ubiquitinated proteins further suggesting impairments of the UPS in PD (Iwatsubo et al., 1996; Lowe et al., 1988).

Mutations in genes such as parkin encoding UPS enzymes are associated with familial PD while knock-out of the $26 \mathrm{~S}$ proteasome in mouse models leads to neurodegeneration and Lewy-like inclusion body formation (Bedford et al., 2008; Dekker et al., 2003; Kitada et al., 1998; Leroy et al., 1998). The $26 \mathrm{~S}$ proteasome consists of a $20 \mathrm{~S}$ proteolytic core particle and two regulatory $19 \mathrm{~S}$ ATPase subunits that form a lid complex. Poly-ubiquitin tagged proteins are recognized by the proteasome leading to selective proteolysis in an ATP-dependent manner. Protein degradation can also occur ubiquitin- and ATP-independently in the $26 \mathrm{~S} / 20 \mathrm{~S}$ proteasome, i.e. as described for $\alpha$-syn (Rott et al., 2011; Tofaris et al., 2001; Voges et al., 1999).

Rodent models of PD have been generated using inhibitors of the UPS - a detailed review of these models can be found in Bentea et al. (2017). One such inhibitor is lactacystin, which spontaneously undergoes hydrolysis to the more potent inhibitor clasto-lactacystin- $\beta$-lactone (Dick et al., 1996). Clasto-lactacystin- $\beta$-lactone can then enter cells, bind covalently to the $20 \mathrm{~S}$ proteasome and irreversibly inhibit its proteolytic sites affecting both the 20S and 26S degradation routes (Dick et al., 1997). Advantages of lactacystin inhibition models are that they reproduce core pathogenic pathways in PD including dopamine cell loss and protein accumulation and aggregation associated with motor impairments (McNaught et al., 2002; Niu et al., 2009; Pienaar et al., 2015). Controversy still exists about the UPS-inhibition models, as some laboratories have failed to produce an animal model of PD after systemic administrations of various inhibitors (Kordower et al., 2006; Mathur et al., 2007) although others have succeeded (McNaught et al., 2004; Schapira et al., 2006).

In contrast, models using direct delivery of UPS inhibitors to either substantia nigra (SN) or the medial forebrain bundle (MFB) have produced more promising and consistent PD-like syndromes 
(Konieczny et al., 2014; Li et al., 2008; Lorenc-Koci et al., 2011; Xie et al., 2010). Intranigral injection of lactacystin has been shown to produce dopaminergic dysfunction evidenced by both reduced midbrain tyrosine hydroxylase $(\mathrm{TH})$ staining and striatal $(+)-\alpha-\left[{ }^{11} \mathrm{C}\right]$ dihydrotetrabenazine $\left(\left[{ }^{11} \mathrm{C}\right] \mathrm{DTBZ}\right)$ uptake on PET imaging (Harrison et al., 2016; Mackey et al., 2013). Radiolabeled DTBZ binds with nanomolar-affinity to the vesicular monoamine transporter 2 (VMAT2) of synaptic vesicles in monoaminergic neurons (Scherman et al., 1988) and can non-invasively track levels of presynaptic dopamine terminals in the striatum (Koeppe et al., 1996). PET imaging using radiolabeled DTBZ has shown VMAT2 reduction in striatum correlating with disease severity of PD patients (Hsiao et al., 2014) and SN cell counts in 1-methyl-4-phenyl-1,2,3,6-tetrahydropyridine (MPTP)-treated primates (Brown et al., 2013). Furthermore, $\left[{ }^{11} \mathrm{C}\right] \mathrm{DTBZ}$ PET has previously shown loss of dopamine neuron integrity in rodent models after 6-hydroxydopamine lesion, UPS inhibition and adeno-associated virus vector induced $\alpha$-syn overexpression (Mackey et al., 2013; Phan et al., 2017; Strome et al., 2006).

We wished to extend these findings to a large animal model, more suitable for behavioural observations and PET imaging. Here, we use a combination of behavioural tests, neuroimaging with $\left[{ }^{11} \mathrm{C}\right] \mathrm{DTBZ}$ and histological examination to assess the effect of inhibiting the UPS with lactacystin in the Göttingen minipig, by unilaterally administering a dose of lactacystin in the MFB of the nigrostriatal pathway. 


\section{Material and methods}

\section{Animals}

The Danish Animal Experiments Inspectorate (2016-15-0201-01138) approved this study and all procedures involving animals were conducted in compliance with the ARRIVE guidelines and the 2010/63/EU directive for animal experiments. Four female Göttingen minipigs from Ellegaard Minipigs ApS (Dalmose, Denmark) aged $10.8 \pm 0.4$ months with an average weight of $21.0 \pm 1.2 \mathrm{~kg}$ were housed two in each cage at the Aarhus University farm. They were fed a restricted diet with free access to tap water. Humidity and temperature were kept at $50-55 \%$ and $20^{\circ} \mathrm{C}$. Animals acclimatized for two weeks prior to the study. On the study days, they were fasted with free access to water.

\section{Surgery}

Animals were pre-medicated with $0.8 \mathrm{mg} / \mathrm{kg}$ midazolam and $6 \mathrm{mg} / \mathrm{kg}$ azaperone IM. Ear veins were catheterized ( $21 \mathrm{G}$ venflon) and anaesthesia was induced with approximately $2.5 \mathrm{mg} / \mathrm{kg}$ propofol IV. Shortly afterwards, the minipigs were intubated $(6.0 \mathrm{~mm})$ and anaesthesia was maintained at approximately $2.1 \%$ isoflurane through a mechanical ventilation setup with $3000 \mathrm{~mL} / \mathrm{min}$ mix of 0.5 part $0_{2}$ and 1 part medical air. Temperature, pulse, oxygen saturation and reflexes were monitored, and animals were kept hydrated with a saline drip and warm with an electric blanket during surgery (Alstrup, 2010).

Prior to fixation in a magnetic resonance image (MRI)-compatible localizer box (Bjarkam et al., 2009), $1.25 \mathrm{mg} / \mathrm{kg}$ of $0.5 \%$ bupivacaine hydrochloride (Marcain ${ }^{\oplus}$, AstraZeneca, DK) was injected subcutaneously over the frontal and zygoma bones at the sites of the surgical incision and at the fixation pin holes. As a reference point, a copper-sulfate filled fiducial marker was placed in a drill hole in the skull close to bregma (Glud et al., 2017). Animals then underwent a 3D T1-weighted MRI brain scan (1.5-T Achieva dStream, Philips) at the MR Research Centre at Aarhus University Hospital with 1x1x1 mm voxels, TE 3.7 ms, TR 2200 ms, TI 960 ms, FA 9 deg, 256 x 256. During the 10 min MRI acquisition, animals were kept anaesthetized with propofol, and hand ventilated.

Based on the reconstructed $1 \times 1 \times 1 \mathrm{~mm}$ image, medio-lateral, anterior-posterior and ventral-dorsal coordinates were calculated relative to the fiducial marker. Coordinates were calculated for three injection sites $1 \mathrm{~mm}$ apart (anterior-posterior) in the MFB (Figure 1). A stereotaxic frame was attached to the localizer box, the skull was opened and an incision was made in the dura (Glud et al., 
2011) to permit descent of the cannula to the predetermined coordinates. A total of $100 \mu \mathrm{g}$ lactacystin (CAS\# 1258004-00-0, Calbiochem) dissolved in $75 \mu \mathrm{L}$ saline $(n=3$, pig\#2,3,4) or saline alone ( $n=1$, pig\# 1 ) was injected unilaterally using a $50 \mu \mathrm{L} 705 \mathrm{RN}$ Hamilton Syringe (VWR) along three anterior-posterior sites $(25 \mu \mathrm{L}$ each) in the nigrostriatal pathway. Infusion rate was $5 \mu \mathrm{L} / \mathrm{min}$ over 4 minutes and at one-minute intervals the needle was retracted $1 \mathrm{~mm}$ to allow diffusion of the solution. The needle was left in place for an additional 3 minutes before withdrawal and the skin was sutured. Postoperatively, the animals received antibiotics and analgesics including $0.03 \mathrm{mg} / \mathrm{kg}$ buprenorphine IM (Temgesic ${ }^{\oplus}$, Indivior UK Limited) three times a day and $1.5 \mathrm{mg} / \mathrm{kg}$ meloxicam (Metacam $^{\circledast}$, Boehringer Ingelheim, DK) and 30,000 IE/kg benzylpenicillin procain (Penovet ${ }^{\oplus}$, Boehringer Ingelheim, DK) once a day for 3 days.

\section{Behavioural analysis}

Behavioural ratings were performed at baseline and every week post-surgery in a $13 \mathrm{~m}^{2}$ arena for 10 min. Videos were acquired at every performance and were reassessed for each individual pig by comparing with the baseline recordings to reduce subjective bias. Neurological scoring of the animals was based on Mikkelsen et al. (1999) and included (A) Motility - 0: normal, 1: slightly compromised, 2: moderately compromised, 3: moderately compromised (freezing behaviour), 4: severely compromised, (B) Position abnormality/Coordination - 0: normal, 1: abnormal leg positioning (i.e. crossing of hindlimbs), 2: balance compensation, 3: clear coordination issues, and (C) Feeding function - 0: normal, 1: compromised, 2: rigid jaws (total maximum score $=9$ ). In addition to the feeding function, we measured a feeding duration obtained by timing the animals as they ate $1.5 \mathrm{dL}$ of food pellets, from snout in until empty.

\section{$\left[{ }^{11}\right.$ C]DTBZ PET}

One week pre-surgery and 3 weeks post-surgery animals had a $\left[{ }^{11} \mathrm{C}\right] \mathrm{DTBZ}$ PET scan. As previously described (Landau et al., 2017), minipigs received a mixture of $1.2 \mathrm{mg} / \mathrm{kg}$ midazolam and $6.0 \mathrm{mg} / \mathrm{kg}$ S-ketamine IM prior to inserting an ear vein catheter through which induction of anaesthesia was administered (mixture of $1.2 \mathrm{mg} / \mathrm{kg}$ midazolam and $3.0 \mathrm{mg} / \mathrm{kg}$ S-ketamine). Animals were then intubated and mechanically ventilated $3000 \mathrm{~mL} / \mathrm{min}$ with an $\mathrm{O}_{2}$ and medical air ratio of 1:2.2 and anaesthesia was maintained with $2.1 \%$ isoflurane. Anaesthetic levels, temperature and vital functions were monitored regularly throughout the scan (Alstrup, 2010). 
Minipigs were placed in a Biograph 64 TruePoint PET/CT scanner (6.7.2, Siemens) with the brain at the centre of the field of view. A transmission CT scan was obtained for attenuation correction. The average injected dose, mass and specific activity at the time of injection of $\left[{ }^{11} \mathrm{C}\right] \mathrm{DTBZ}$ were $385.7 \pm 19.9 \mathrm{GBq}, 2.1 \pm 2.0 \mu \mathrm{g}$ and $64.8 \pm 39.7 \mathrm{GBq} / \mu \mathrm{mol}$, respectively. No statistically significant differences in these were observed between the baseline and the post-surgery scan values. $\left[{ }^{11} \mathrm{C}\right] \mathrm{DTBZ}$ was dissolved in $20 \mathrm{~mL}$ saline and injected intravenously over $30 \mathrm{~s}$ followed by a $20 \mathrm{~mL}$ saline flush (Alstrup et al., 2017, in press). PET was acquired for $90 \mathrm{~min}$ in list mode. Images were reconstructed using TrueX with a point-spread function ( 3 iterations and 21 subsets) and smoothed with a $2 \mathrm{~mm}$ Gaussian kernel. PET data were rebinned as: 5x1, 3x5, 4x10 and 2x15 min time frames.

\section{Data analysis}

For quantitative analysis, reconstructed images were processed in PMOD v. 3.610 (PMOD Technologies, Zürich, Switzerland). Images were rigidly co-registered to a minipig average MRI with standard volumes of interest (Watanabe et al., 2001). Time-activity curves were obtained from right and left striatum and the cerebellum. Using the Logan graphical approach (Logan et al., 1996), the slope of data from 20-90 minutes was used to calculate the distribution volume ratio (DVR) in a target region of interest relative to the cerebellum (as a low dopamine terminal-containing reference). The average tissue-to-plasma rate constant from the reference region $\left(k_{2}{ }^{\prime}\right)$ did not differ between the animals and a fixed value $\left(0.15 \mathrm{~min}^{-1}\right)$ was used for all. Data are presented as the nondisplaceable binding potential $\left(B P_{N D}=D V R-1\right)$ using Graphpad Prism v7.0. Parametric maps of Logan reference $B P_{N D}$ were calculated from the co-registered PET images in the PXMOD toolbox.

\section{Histology analysis}

One month post-surgery, minipigs were deeply anaesthetized with a mixture of $2.5 \mathrm{mg} / \mathrm{kg}$ midazolam and $20 \mathrm{mg} / \mathrm{kg}$ azaperone and were euthanized with an overdose of $20 \mathrm{~mL} 400 \mathrm{mg} / \mathrm{mL}$ pentobarbital sodium (Exagon ${ }^{\oplus}$ Vet, Richter Pharma, Austria) transcutaneous to the heart before transcardial perfusion with $5 \mathrm{~L}$ of $4 \%$ paraformaldehyde was performed (Ettrup et al., 2011). The fixed brain was removed for histological analysis as described in Bjarkam et al. (2017). Brains were stored in $4 \%$ formaldehyde at $4^{\circ} \mathrm{C}$ until they were pre-cut into $1 \mathrm{~cm}$ thick coronal blocks and cryoprotected in 30\% sucrose and 0.15 M Sørensen's phosphate buffer for one week. Brain slabs were frozen in isopentane cooled to $-40^{\circ} \mathrm{C}$ for $1 \mathrm{~min}$ before being cut into coronal sections $(40 \mu \mathrm{m})$ on a CryoStar NX70 (Thermo Scientific). Every brain slab covering striatum, thalamus, SN and locus coeruleus was cut in 10 series (slices in each series were spaced $400 \mu \mathrm{m}$ ) and were stored free- 
floating in DeOlmos at $-20^{\circ} \mathrm{C}$. One set was mounted directly on $0.5 \%$ gelatin-coated glass and stained with Nissl. In brief, mounted sections were stained with $0.1 \%$ toluidine blue in citrate buffer ( $\mathrm{pH} 4.0$ ) at room temperature for $9 \mathrm{~min}$, rinsed shortly in distilled water, dehydrated $2 \times 30 \mathrm{sec}$ in 99\% alcohol, cleared with xylene and cover slipped with Eukitt ${ }^{\oplus}$.

On day 1 of the immunohistochemistry staining, free-floating sections were initially washed in trisbuffered saline containing 1\% Triton X-100 (TBS-T, $0.05 \mathrm{M}$, pH 7.4) for 3 x 5 min. Target retrieval was conducted for all antibodies, except anti- $\alpha$-syn, in a water bath at $80^{\circ} \mathrm{C}$ with Dako retrieval buffer (S1699) for $30 \mathrm{~min}$ followed by cooling to room temperature for $30 \mathrm{~min}$. Retrieval buffer was washed off with $2 \times 5$ min TBS-T and TBS was added for 5 min prior to quenching the endogenous peroxidase with $3 \% \mathrm{H}_{2} \mathrm{O}_{2}$ in $0.05 \mathrm{M}$ TBS for 15 minutes. Sections were again rinsed in TBS for 10 min followed by 2 x 10 min in TBS-T. Then, sections were incubated with $0.2 \%$ skim milk powder in TBS-T for 30 minutes. Finally, rabbit anti-TH (1:500, \#AB152, Lot: 2893306, EDM Millipore Corp.), mouse anti-HLA-DR (1:100, \#M0746, Lot: 20020039, Dako), or mouse anti- $\alpha$-syn (1:5000, Syn211, \#MA1-12874, Lot: QF2033452, Thermo Scientific) in the $0.2 \%$ milk solution was added as primary antibody to the slides and incubated overnight at $4^{\circ} \mathrm{C}$. Primary antibody was omitted for a few sections for every procedure, which served as negative controls.

On day 2, sections were rinsed in $3 \times 10$ min TBS-T followed by incubation for 2 hours with the appropriate horseradish peroxidase (HRP) labeled secondary antibody (anti-TH, 1:400, HRPlabeled anti-rabbit, \#P0448, Lot: 20017525 Dako or anti-HLA-DR, 1:400, HRP-labeled anti-mouse, \#P0447, Lot: 20019698, Dako) or 1 hour with biotinylated secondary antibody (anti- $\alpha$-syn 211, 1:200, biotinylated anti-mouse, \#A90-116B, Lot: A90-116B-10, Bethyl) in TBS-T. Sections were rinsed again 3 times in TBS-T for $10 \mathrm{~min}$. For the biotin-labeled secondary antibody (anti- $\alpha$-syn), an avidin-biotin-peroxidase complex solution (Vectastain ${ }^{\oplus}$ elite $^{\oplus}$ ABC-kit, \#VECTPK-6100, Vector Laboratories) was added for 1 hour prior to a final $10 \min 2 \times$ TBS-T and $1 \mathrm{x}$ TBS rinse. The bound HRP or avidin-biotin complex were visualized with a mix of $0.1 \% 3,3^{\prime}$-diaminobenzidine (DAB; DAB-Safe, Saveen Werner AB) in TBS and $0.03 \% \mathrm{H}_{2} \mathrm{O}_{2}$. After $5 \mathrm{~min}$, the reaction was terminated by a $3 \times 1 \mathrm{~min}$ in TBS. Sections were then mounted on $0.5 \%$ gelatin-coated glass slides, dehydrated in 2 x 5 min 99\% ethanol and cleared in xylene for 5 min before being coverslipped using Eukitt ${ }^{\oplus}$. Brain sections were photographed using a Leica camera (DFC 480) connected to a Leica microscope (DM5000B). 


\section{Analysis of TH immunoreactivity in SN and VTA}

TH immunohistochemistry staining was semi-quantified by selecting matched sections of the SN and VTA of each minipig and measuring the area covered by DAB product presented in $\mu \mathrm{m}^{2}$. Please note that we did not measure the DAB staining intensity, because DAB does not meet the LambertBeer criteria for photometric material, as there is no linear association between staining intensity and $\mathrm{DAB}$ concentration. First, a series of 24 bit colour photographs was taken with the Leica camera and microscope using similar exposition time, levels and white balance (2560 x 1920 pixels). Then, the pictures were merged in Photoshop (Adobe, version 16.0.0, 2015), to obtain a final coronal section of the brain. To allow measurements, photographed sections were converted into 8 bit images followed by semi-automatic segmentation (threshold) using intensity channel from HSI images, which gives the highest contrast between background and stained tissue. After the initial automatic setting, the threshold was manually corrected to ensure that all TH-positive stains were covered within SN and VTA. The area $\left(\mu \mathrm{m}^{2}\right)$ was estimated in ImageJ (Schneider et al., 2012) and averaged per hemisphere/brain for each minipig. As SN and the VTA are difficult to distinguish, the regions were carefully delineated.

\section{Results}

\section{Behaviour}

The minipig injected unilaterally with saline (pig \#1) displayed no feeding or behavioural changes in the neurological scoring test compared to its own baseline scores (Figure $2 \mathrm{a} \& \mathrm{~b}$ ). Pig\#2 unilaterally injected with lactacystin displayed clear coordination issues, which were most predominant in the contralateral hind-limb post-surgery. Small steps, rigidity and midline positioning of both hind limbs characterized its gait, the latter a consequence of the ipsilateral hind limb compensating for the contralateral leg rigidity. Its motility was moderately compromised with freezing behaviour especially in the first 3 weeks following the unilateral lactacystin injection (Figure 2a). Pig\#2 also displayed the most pronounced change in eating behaviour, with slow eating speed and rigid jaws (Figure 2b). Pig\#3 had mildly impaired mobility over the 4 weeks compared to its baseline assessment with an increased eating duration the first week (Figure 2a \& b). Pig\#4 displayed substantial spontaneous rotational behaviour and freezing following the unilateral lactacystin injection, which was still present 4 weeks after injection. The animal rotated towards the contralateral injection site in small circles with the hind limbs placed in the centre only moving slightly, while the front limbs moved on the perimeter of the circle. It showed only small changes in eating behaviour and mobility compared to its baseline status (Figure 2a \& b). 


\section{$\left[{ }^{11}\right.$ C]DTBZ PET results}

We imaged dopaminergic terminal function in vivo with $\left[{ }^{11} \mathrm{C}\right] \mathrm{DTBZ}$ PET, a marker of VMAT2 availability in the minipig brain at baseline and following unilateral injection of saline or lactacystin in the nigrostriatal pathway. Binding of the tracer to the contralateral and ipsilateral striatum at baseline was similar and consistent between the 4 animals. Quantification of $\left[{ }^{11} \mathrm{C}\right] \mathrm{DTBZ} B P_{N D}$

revealed an average of $-36 \pm 8 \%\left(\%\left(\frac{\text { post }}{\text { baseline }}-1\right)\right)$ reduction in binding to the VMAT2 in the ipsilateral striatum across all 3 lactacystin-injected minipigs while the saline-injected minipig showed only a small decline (-10\%) in binding at three weeks compared to their own ipsilateral striatum baseline values (Figure 3a). In the post intervention scan, the VMAT2 binding to the contralateral striatum was unchanged for the saline-injected pig\#1 and the lactacystin-injected pig\#2 compared to their own baseline. In contrast, a decreased binding of $-25 \pm 3 \%$ was observed in the contralateral striatum for pigs \#3 and \#4 compared to their own baseline binding.

By comparing the \%ipsilateral vs. contralateral change of each minipig $\left(\% \frac{\text { ipsilateral }}{\text { contralateral }}\right)$, we observed no change in binding at baseline or after three weeks for the saline-injected pig\#1. Asymmetric binding was however observed for all 3 lactacystin-injected minipigs with ipsilateral binding being lowered to an average of $76 \pm 14 \%$ of contralateral binding at the three week postinjection PET scan (Figure 3b).

In figure $3 c$, PET images are presented as $B P_{N D}$ voxel-wise maps and asymmetric binding in striatum of the three lactacystin-injected minipigs can be seen. Moreover, it should be noted that the average binding in both the contralateral and ipsilateral striatum of the lactacystin-injected minipigs appear lower than both the binding seen in the baseline and saline scans.

\section{Histology}

The Nissl stained brain sections and TH stains of the fiber tract confirm that the MFB in the nigrostriatal pathway was correctly targeted. Targeting was similar for all minipigs anterior to the SN and ventral to thalamus, though in pig\#3 the injection seemed to be located slightly dorsal to the MFB. The Nissl stains of SN pars compacta $(\mathrm{SNc})$ showed that fewer neurons were visualized in the ipsilateral side compared to the contralateral side in the lactacystin-injected animals, especially in 
pig\#2 and \#4 (Figure 4b). In the saline-injected pig\#1, SNc of both hemispheres appeared identical (Figure 4b).

Brain sections covering the SN from all 4 animals were stained with antibodies specific for the $\mathrm{TH}$ enzyme localized in the cytosol of dopaminergic neurons (Figure 5a). The three lactacystin-injected minipigs had asymmetrical changes in the $\mathrm{SN}$, which was in contrast to the saline-injected pig\#1 that displayed symmetrical anti-TH staining in the SN. We semi-quantified this difference by measuring the nigral TH-immunopositive area in both sides of all minipigs and an average reduction of $-62 \% \pm 25 \%$ for the lactacystin-injected pigs was found in the ipsilateral side compared to the contralateral side while a $10 \%$ increase was found in the saline-injected minipig (Figure $5 \mathrm{~b}$ ). In contrast, the area of TH-immunoreactivity in the ventral tegmental area (VTA) close to the midline on the ipsilateral side was not affected by lactacystin administration and no differences were found between the ipsilateral and contralateral side in any of the animals or between the saline- and lactacystin-injected minipigs (Figure 5b).

Similar staining patterns were observed in the striatum, suggesting a loss of TH positive projections to the ipsilateral striatum in the animals injected with lactacystin (Figure 5c). No differences between the two hemispheres were observed in striatum of the saline-injected minipig. Brain sections covering locus coeruleus were also investigated for TH-immunoreactivity, however, homogenous expression was observed in both hemispheres of all minipigs.

Lactacystin injection in the nigrostriatal pathway caused an inflammatory response, which manifested as increased HLA-DR expression by activated microglia in the SN, VTA and other areas adjacent to the needle of the lactacystin-injected minipigs (Figure 6). For all 4 injected minipigs, including the saline-injected minipig, local microglial activation was observed close to the needle tract progressing dorsally from the cortex and ventrally through the central telencephalic white matter tract and thalamus reaching the MFB. Furthermore, microglial activation was also seen in the ipsilateral striatum (predominantly the internal capsule), thalamus and in the SN away from the injection site in the lactacystin-injected minipigs. Most microglia observed in the different brain regions were hypertrophic and in the ipsilateral SN of the lactacystin-injected animals, some were amoeboid with enlarged cell bodies and thick processes characteristic of highly activated microglia (Figure 6). Only a few microglia were observed in the saline-injected minipig on the ipsilateral side, at a distance from the injection site (striatum, SN and VTA), and most had a resting morphology. However, some light hypertrophic microglia were observed locally in the ipsilateral VTA. Similarly, 
only lightly scattered microglia with a ramified morphology, and a few with hypertrophy, were observed contralateral to the injection site in all minipigs (Figure 6).

In addition to the TH and HLA-DR stains, we also examined adjacent slices in all animals for possible $\alpha$-syn aggregates in the SN and VTA. In the SNc, mostly fibers stained for $\alpha$-syn were visible in all the minipigs, although $\alpha$-syn-immunopositive intracytoplasmatic granular staining could be observed in some neurons in the ipsilateral side of the lactacystin-injected minipigs (Figure 7). In $\mathrm{SN}$ pars reticulata ( $\mathrm{SNr}$ ), a punctate and granular staining of $\alpha$-syn was observed in all pigs, though it appeared slightly denser with larger granules in the lactacystin-injected minipigs compared to the saline-injected minipig (Figure 7). No differences were observed between the ipsilateral and contralateral side in any of the animals.

The neurons located in the ipsilateral VTA showed a clear expression of $\alpha$-syn in the soma (Figure 7). This was in contrast to the site contralateral to the injection, where only puncta, presumably synapses, were visible in the VTA. Interestingly, the accumulated $\alpha$-syn in neurons was not only seen in all 3 lactacystin-injected minipigs, but also in the ipsilateral side of the saline-injected minipig (Figure 7). This indicated that the needle insertion, including transient rupture of the blood-brain barrier, and the tension caused by the volume of fluid injected in the MFB affected $\alpha$ syn turnover in primarily VTA located neurons.

\section{Discussion}

This study shows that inhibition of the UPS by unilateral lactacystin injection induces a hemiparkinsonian-like phenotype in Göttingen minipigs. This phenotype is associated with behavioural slowness and asymmetry, in vivo reductions in striatal $\left[{ }^{11} \mathrm{C}\right] \mathrm{DTBZ}$ binding detected by PET, and TH binding asymmetry in striatum and SN, HLA-DR-positive microglia and $\alpha$-syn positive neurons in SN detected by histology. For the first time, we translate the UPS inhibition animal model mimicking Parkinson's disease to the Göttingen minipig.

An advantage of using a large animal model like the Göttingen minipig is that it has a large brain with clearly defined gyri, which makes it suited for neuroimaging deep brain structures using human scanners at sufficient resolution. Another advantage is the ability to use human clinical devices and stimulation parameters for interventions, for example for deep brain stimulation, in future studies. As previous studies had found nigral dopamine neuron loss, $\alpha$-syn accumulation, 
glial activation and motor deficits following stereotaxic infusion of lactacystin in the MFB of rats and mice (Li et al., 2008; Vernon et al., 2011; Xie et al., 2010), we also decided to inject the minipigs in the MFB to increase our chances of reaching a maximum number of SN neurons via the dopaminergic fibers while avoiding direct harm to the SN neurons.

We observed bradykinesia and rigidity in the contralateral limbs of two minipigs and spontaneous contralateral rotations in another minipig following the unilateral injection of lactacystin in the nigrostriatal pathway, which lasted throughout the 4 weeks of this study (Figure 2a). Spontaneous rotations were only observed in one animal, which might be a result of differential lesion severity across the animals. The contralateral hypokinesia and the need to maintain balance may play a part in the observed contralateral rotations in one minipig. Also, as has been speculated for both lactacystin- and epoxomicin-injected rodents, the spontaneous contralateral rotations might have been caused by hyperactivity of the surviving dopaminergic neurons (Bentea et al., 2015; Konieczny et al., 2016; Subramaniam et al., 2014). Indeed, other studies have shown unpredictable rotation behaviour in unilaterally-lesioned animals in response to L-dopa due to uncontrolled fluctuations in extracellular dopamine varying with lesion degree (Abercrombie et al., 1990; Kaariainen et al., 2012).

Our finding of decreased $\left[{ }^{11} \mathrm{C}\right] \mathrm{DTBZ} B P_{N D}$ in the ipsilateral striatum of lactacystin-injected minipigs is consistent with human studies of decreased binding potential in striatum of PD patients compared to normal controls (Bohnen et al., 2006) as well as in various animal models in rodents and primates (Brown et al., 2013; Mackey et al., 2013; Phan et al., 2017). Specifically, in clinically hemi-parkinsonian patients, decreased striatal $\left[{ }^{11} \mathrm{C}\right] \mathrm{DTBZ}$ binding was noted in the striatum contralateral to the most affected or symptomatic side (Lee et al., 2000). Even though VMAT2 is present in both noradrenergic, serotonergic and dopaminergic neurons, the striatal content of dopaminergic neurons represents $\sim 90 \%$ of $\left[{ }^{11} \mathrm{C}\right]$ DTBZ binding. Another human study by Hsiao et al. (2014) detected a decreased binding in PD patients using $\left[{ }^{18} \mathrm{~F}\right] \mathrm{DTBZ}$ PET both in striatum and SN correlating with disease stage. While clear decreased VMAT2 binding was detected in the striatum in our study, we were unable to accurately localize and detect any changes of the minipig SN using $\left[{ }^{11} \mathrm{C}\right]$ DTBZ PET due to the size of the SN/VTA area and partial volume effects associated with poor scanner resolution. However, a good correlation was found between $\left[{ }^{11} \mathrm{C}\right] \mathrm{DTBZ}$ striatal binding and nigral cell counts in MPTP-treated non-human primates (Brown et al., 2013). 
A small decline in $\left[{ }^{11} \mathrm{C}\right] \mathrm{DTBZ}$ binding was noted in the ipsilateral striatum (10\%) of the saline treated minipig three weeks after injection compared to its baseline value but its \% ipsilateral/contralateral ratio remained at $\sim 100 \%$ post-surgery. Furthermore, no visual differences were detected in its immunohistochemistry in either the SN or the striatum thus this small difference may be accounted for by a number of factors such as test-retest reproducibility and positioning of the animal. Lactacystin-injected pigs \#2, \#3 and \#4 had similar reduced ipsilateral striatal $\left[{ }^{11} \mathrm{C}\right] \mathrm{DTBZ} B P_{N D}$ binding ( 36\%) compared to baseline. When assessing nigral TH levels by immunohistochemistry, we observed a larger reduction in pigs \#2 and \#4 (73 and 90\%, respectively) in the ipsilateral vs. contralateral SN, whereas pig \#3 showed a lesser decline of $31 \%$. Small deviations in placement of the needle may have contributed to the differing severity of lesion and symptoms between animals. The lactacystin injection seemed to be located slightly above the MFB in pig\#3 on the histological sections, and indeed, pig\#3 presented with fewer motor symptoms and larger ipsilateral TH positive area than pig\#2 and \#4. Naturally, small inter-individual effects may influence the site of injection, as the stereotaxic coordinates are calculated from a MR resolution of $1 \times 1 \times 1 \mathrm{~mm}$ and the needle may bend slightly during the penetration of 3-4 cm brain tissue from the cortical surface to the MFB target. Furthermore, the precision of the stereotactic frame may introduce minor errors.

The average percentage ipsilateral to contralateral reduction in the $\left[{ }^{11} \mathrm{C}\right] \mathrm{DTBZ} B P_{N D}$ was not as large as the ipsilateral side change at baseline vs. three weeks after lactacystin-injection (Figure $3 \mathrm{~b}$ ) due to the contralateral decreased binding in striatum present in pig\#3 and \#4. This suggests that the contralateral side was also affected by the lactacystin injection three weeks after the surgery presumably due to hemisphere interconnections or passive diffusion. VMAT2 is believed to be a stable terminal density marker and to not undergo major compensatory regulatory changes (Suzuki et al., 2001; Vander Borght et al., 1995), however, this assumption has been challenged (Kilbourn et al., 2010; Tong et al., 2008). Decreased VMAT2 binding could, therefore, be due to increased vesicular dopamine levels, presynaptic dopamine terminal impairment or cell death of the SN neurons. The histology images (Figure 5) show a visible loss of TH-positive neurons in the ipsilateral SN and of TH-positive fibers in the striatum of the lactacystin-injected minipigs, which reflect either down-regulation of the TH enzyme or actual cell loss. Most likely, the observed ipsilateral VMAT2 and TH reductions are a mix of both scenarios (cell loss and enzyme/transporter reduction), and the Nissl stains (Figure $4 \mathrm{~b}$ ) as well as the motor symptoms support that cell loss 
occurred. Intact TH-stains of the contralateral SN and striatum was observed in all 3 lactacystininjected minipigs.

It has to be noted that a direct comparison between $\mathrm{TH}$ staining and $\left[{ }^{11} \mathrm{C}\right] \mathrm{DTBZ}$ imaging cannot be done, as the $\left[{ }^{11} \mathrm{C}\right] \mathrm{DTBZ}$ PET imaging was done 3 weeks after injection while the animals were euthanized 4 weeks after injection providing tissue for the histology. However, we can conclude that already after 3 weeks, synaptic disruption was seen in the asymmetrical in vivo striatal $\left[{ }^{11} \mathrm{C}\right] \mathrm{DTBZ}$ binding, which was still present 4 weeks after injection visualized by TH staining.

Post-mortem human studies and animal experiments have found glial-mediated neuroinflammation to be associated with PD pathogenesis, in addition to $\alpha$-syn pathology (Hirsch and Hunot, 2009; McGeer and McGeer, 2008). In our model, we observed ipsilateral HLA-DRpositive microglia in the SN and VTA of the three lactacystin-injected minipigs (Figure 6). HLA-DR is an antigen presenting major histocompatibility complex (MHC) class II receptor that is upregulated when microglia are activated. The inflammatory response in the saline-injected minipig was localized close to the needle tract, and as only few light hypertrophic microglia were observed in the ipsilateral VTA, it is likely that it was solely due to the minor cell damage caused by the needle and fluid injection.

On the contrary, lactacystin induced scattered microglia activation in the SN and VTA ipsilaterally, while only few HLA-DR positive glial cells were observed contralaterally. Microglial activation following lactacystin administration has previously been observed in the SN of rodents and may reflect pro- or anti-inflammatory effects leading to protection against or increased neuronal degeneration depending on multiple factors such as doses, proximity and timing (Pienaar et al., 2015; Xie et al., 2010). We followed the minipigs for only 4 weeks so it is uncertain if the activation would have sustained for a longer time. Interestingly, monkeys injected with MPTP showed dopaminergic neurodegeneration and persistent microglial activation several years after intoxication further suggesting microglial activation is involved in the neurodegeneration-process (McGeer et al., 2003).

Based on the TH-immunoreactivity, the dopamine neurons in the VTA were not affected by the lactacystin-injection (Figure 5). This insensitivity of the VTA to lactacystin has previously been observed in other animal models (Bentea et al., 2015; Mackey et al., 2013). This is consistent with a 
study in mice infused directly in the VTA with a proteasome inhibitor, which showed no functional changes in VTA dopaminergic neurons while the nigral neurons were harmed (Subramaniam et al., 2014). Interestingly, we observed that $\alpha$-syn accumulated in the ipsilateral VTA neurons and to a lesser extent in the SNc neurons in both lactacystin- and saline-injected minipigs, while synapses were stained more widespread in both sides (Figure 7). This indicated that the needle penetration and volume of fluid itself affected either the degradation system or may have led to increased expression of $\alpha$-syn in the VTA located neurons. Hence it is a non-specific effect unrelated to lactacystin. Light hypertrophic microglia were observed in the ipsilateral VTA of both the salineand lactacystin-injected minipigs, which were not present contralaterally. Thus, it can be speculated that the minor inflammatory response occurring in VTA could trigger $\alpha$-syn accumulation, as MHC class II expression assessed by HLA-DR has been shown to significantly correlate with deposition of $\alpha$-syn in SN of human PD patients (Croisier et al., 2005).

Previous reports have shown an increased ipsilateral SNr $\alpha$-syn immunoreactivity to lactacystintreatment in both mice and rats compared to control animals accompanied by neuroinflammation and oxidative stress (Miwa et al., 2005; Savolainen et al., 2017). We observed immunopositive $\alpha$-syn granules in both the ipsilateral and contralateral SNr that were slightly more pronounced in the lactacystin- than the saline-injected animals (Figure 7), presumably caused by the minor inflammatory response seen in these animals.

The main limitations of this study are the lack of quantitative stereology and small sample sizes as only three animals were injected with lactacystin and only one with saline as a control. However, all three lactacystin animals showed consistent behavioural, neuropathological and neurotransmission effects, so we conclude that lactacystin has similar effects in minipigs as it has in rodents. Moreover, although we only had one saline control animal, we have used the $\left[{ }^{11} \mathrm{C}\right] \mathrm{DTBZ}$ tracer in a number of other studies in Göttingen minipig and the binding values of our saline treated animal at both timepoints are consistent with the findings in untreated animals in our previous work (Lillethorup et al., unpublished results). Furthermore, by acquiring baseline behaviour and PET data and injecting unilaterally, the animals became their own internal controls for the longitudinal assessment.

Similar UPS inhibition models in rodents have reported non-specific damage of lactacystin to nondopaminergic and GABAergic neurons in the nigra (Konieczny et al., 2015) as well as to other neighbouring systems such as pedunculo-pontine nucleus (Pienaar et al., 2015) and an additional 
limitation of our study is that other transmitter systems were not investigated here. As lactacystin is an irreversible inhibitor, it would also be interesting to test its effect over a longer time period than the 4 weeks in this study, in a longitudinal design to investigate if the impairments are sustained or lead to even further progression or recovery. However, a recent study suggests that lactacystin induces a stable lesion in rodents during an 8 week period (Mejias et al., 2016) and thus our timeline was likely sufficient for a preliminary study.

\section{Conclusions}

This non-primate large animal injected with lactacystin directly into the nigrostriatal pathway may provide a promising model of PD. Its use is supported by the associated motor impairments, lowered VMAT2 binding in striatum measured by $\left[{ }^{11} \mathrm{C}\right] \mathrm{DTBZ}$ PET, reduced area of TH-positive neurons in $\mathrm{SNc}$ and microglial activation.

\section{Acknowledgement}

The authors thank staff at the Aarhus University Farm (Påskehøjgaard) for their animal care, Michael Pedersen and Steffen Ringgaard for technical assistance at the MR research Centre at Aarhus University Hospital and the Center for Experimental Neuroscience (CENSE) at the Danish Neuroscience Center for their help in coordinating this project.

\section{Funding}

This work was funded by grants from the Bjarne Saxhof Fund administered through the Danish Parkinsonforeningen to AML.

\section{Declaration of interest}

Declarations of interest: none.

\section{References}

Abercrombie, E.D., Bonatz, A.E., Zigmond, M.J., 1990. Effects of L-dopa on extracellular dopamine in striatum of normal and 6-hydroxydopamine-treated rats. Brain Res 525, 36-44.

Alstrup, A.K.O., 2010. Anaesthesia and Analgesia in Ellegaard Göttingen Minipigs. Ellegaard, Denmark.

Alstrup, A.K.O., Munk, O.L., Landau, A.M., Lillethorup, T.P., 2017. PET radioligand injection for pig neuroimaging. Scandinavian Journal of Laboratory Animal Science, in press 
Bedford, L., Hay, D., Devoy, A., Paine, S., Powe, D.G., Seth, R., Gray, T., Topham, I., Fone, K., Rezvani, N., Mee, M., Soane, T., Layfield, R., Sheppard, P.W., Ebendal, T., Usoskin, D., Lowe, J., Mayer, R.J., 2008. Depletion of $26 \mathrm{~S}$ proteasomes in mouse brain neurons causes neurodegeneration and Lewy-like inclusions resembling human pale bodies. J Neurosci $28,8189-8198$.

Bentea, E., Van der Perren, A., Van Liefferinge, J., El Arfani, A., Albertini, G., Demuyser, T., Merckx, E., Michotte, Y., Smolders, I., Baekelandt, V., Massie, A., 2015. Nigral proteasome inhibition in mice leads to motor and non-motor deficits and increased expression of Ser 129 phosphorylated alpha-synuclein. Front Behav Neurosci 9, 68.

Bentea, E., Verbruggen, L., Massie, A., 2017. The Proteasome Inhibition Model of Parkinson's Disease. J Parkinsons Dis 7, 31-63.

Betarbet, R., Sherer, T.B., Greenamyre, J.T., 2005. Ubiquitin-proteasome system and Parkinson's diseases. Exp Neurol 191, S17-27.

Bjarkam, C.R., Cancian, G., Glud, A.N., Ettrup, K.S., Jorgensen, R.L., Sorensen, J.C., 2009. MRI-guided stereotaxic targeting in pigs based on a stereotaxic localizer box fitted with an isocentric frame and use of SurgiPlan computer-planning software. J Neurosci Methods 183, 119-126.

Bjarkam, C.R., Glud, A.N., Orlowski, D., Sorensen, J.C., Palomero-Gallagher, N., 2016. The telencephalon of the Gottingen minipig, cytoarchitecture and cortical surface anatomy, Brain Struct Funct.

Bjarkam, C.R., Orlowski, D., Tvilling, L., Bech, J., Glud, A.N., Sorensen, J.H., 2017. Exposure of the Pig CNS for Histological Analysis: A Manual for Decapitation, Skull Opening, and Brain Removal, J Vis Exp.

Bohnen, N.I., Albin, R.L., Koeppe, R.A., Wernette, K.A., Kilbourn, M.R., Minoshima, S., Frey, K.A., 2006. Positron emission tomography of monoaminergic vesicular binding in aging and Parkinson disease. J Cereb Blood Flow Metab 26, 1198-1212.

Brown, C.A., Karimi, M.K., Tian, L., Flores, H., Su, Y., Tabbal, S.D., Loftin, S.K., Moerlein, S.M., Perlmutter, J.S., 2013. Validation of midbrain positron emission tomography measures for nigrostriatal neurons in macaques. Ann Neurol 74, 602-610.

Croisier, E., Moran, L.B., Dexter, D.T., Pearce, R.K., Graeber, M.B., 2005. Microglial inflammation in the parkinsonian substantia nigra: relationship to alpha-synuclein deposition. J Neuroinflammation 2, 14 .

Cuervo, A.M., Stefanis, L., Fredenburg, R., Lansbury, P.T., Sulzer, D., 2004. Impaired degradation of mutant alpha-synuclein by chaperone-mediated autophagy. Science 305, 1292-1295.

Dekker, M.C., Bonifati, V., van Duijn, C.M., 2003. Parkinson's disease: piecing together a genetic jigsaw. Brain 126, 1722-1733.

Dick, L.R., Cruikshank, A.A., Destree, A.T., Grenier, L., McCormack, T.A., Melandri, F.D., Nunes, S.L., Palombella, V.J., Parent, L.A., Plamondon, L., Stein, R.L., 1997. Mechanistic studies on the inactivation of the proteasome by lactacystin in cultured cells. J Biol Chem 272, 182-188.

Dick, L.R., Cruikshank, A.A., Grenier, L., Melandri, F.D., Nunes, S.L., Stein, R.L., 1996. Mechanistic studies on the inactivation of the proteasome by lactacystin: a central role for clasto-lactacystin beta-lactone. J Biol Chem 271, 7273-7276.

Ebrahimi-Fakhari, D., Cantuti-Castelvetri, I., Fan, Z., Rockenstein, E., Masliah, E., Hyman, B.T., McLean, P.J., Unni, V.K., 2011. Distinct roles in vivo for the ubiquitin-proteasome system and the autophagylysosomal pathway in the degradation of alpha-synuclein. J Neurosci 31, 14508-14520. 
Ettrup, K.S., Glud, A.N., Orlowski, D., Fitting, L.M., Meier, K., Soerensen, J.C., Bjarkam, C.R., Alstrup, A.K., 2011. Basic surgical techniques in the Gottingen minipig: intubation, bladder catheterization, femoral vessel catheterization, and transcardial perfusion, J Vis Exp.

Glud, A.N., Bech, J., Tvilling, L., Zaer, H., Orlowski, D., Fitting, L.M., Ziedler, D., Geneser, M., Sangill, R., Alstrup, A.K.O., Bjarkam, C.R., Sorensen, J.C.H., 2017. A fiducial skull marker for precise MRI-based stereotaxic surgery in large animal models. J Neurosci Methods 285, 45-48.

Glud, A.N., Hedegaard, C., Nielsen, M.S., Soorensen, J.C., Bendixen, C., Jensen, P.H., Mogensen, P.H., Larsen, K., Bjarkam, C.R., 2011. Direct MRI-guided stereotaxic viral mediated gene transfer of alphasynuclein in the Gottingen minipig CNS. Acta Neurobiol Exp (Wars) 71, 508-518.

Harrison, I.F., Anis, H.K., Dexter, D.T., 2016. Associated degeneration of ventral tegmental area dopaminergic neurons in the rat nigrostriatal lactacystin model of parkinsonism and their neuroprotection by valproate. Neurosci Lett $614,16-23$.

Hirsch, E.C., Hunot, S., 2009. Neuroinflammation in Parkinson's disease: a target for neuroprotection? Lancet Neurol 8, 382-397.

Hsiao, I.T., Weng, Y.H., Hsieh, C.J., Lin, W.Y., Wey, S.P., Kung, M.P., Yen, T.C., Lu, C.S., Lin, K.J., 2014. Correlation of Parkinson disease severity and 18F-DTBZ positron emission tomography. JAMA Neurol 71, 758-766.

Iwatsubo, T., Yamaguchi, H., Fujimuro, M., Yokosawa, H., Ihara, Y., Trojanowski, J.Q., Lee, V.M., 1996. Purification and characterization of Lewy bodies from the brains of patients with diffuse Lewy body disease. Am J Pathol 148, 1517-1529.

Kilbourn, M.R., Butch, E.R., Desmond, T., Sherman, P., Harris, P.E., Frey, K.A., 2010. In vivo [11C] dihydrotetrabenazine binding in rat striatum: sensitivity to dopamine concentrations. Nucl Med Biol $37,3-8$.

Kitada, T., Asakawa, S., Hattori, N., Matsumine, H., Yamamura, Y., Minoshima, S., Yokochi, M., Mizuno, Y., Shimizu, N., 1998. Mutations in the parkin gene cause autosomal recessive juvenile parkinsonism. Nature 392, 605-608.

Koeppe, R.A., Frey, K.A., Vander Borght, T.M., Karlamangla, A., Jewett, D.M., Lee, L.C., Kilbourn, M.R., Kuhl, D.E., 1996. Kinetic evaluation of [11C]dihydrotetrabenazine by dynamic PET: measurement of vesicular monoamine transporter. J Cereb Blood Flow Metab 16, 1288-1299.

Konieczny, J., Czarnecka, A., Kaminska, K., Lenda, T., Nowak, P., 2015. Decreased behavioral response to intranigrally administered GABAA agonist muscimol in the lactacystin model of Parkinson's disease may result from partial lesion of nigral non-dopamine neurons: comparison to the classical neurotoxin 6-OHDA. Behav Brain Res 283, 203-214.

Konieczny, J., Czarnecka, A., Lenda, T., Kaminska, K., Lorenc-Koci, E., 2014. Chronic L-DOPA treatment attenuates behavioral and biochemical deficits induced by unilateral lactacystin administration into the rat substantia nigra. Behav Brain Res 261, 79-88.

Konieczny, J., Lenda, T., Czarnecka, A., 2016. Early increase in dopamine release in the ipsilateral striatum after unilateral intranigral administration of lactacystin produces spontaneous contralateral rotations in rats. Neuroscience 324, 92-106.

Kordower, J.H., Kanaan, N.M., Chu, Y., Suresh Babu, R., Stansell, J., 3rd, Terpstra, B.T., Sortwell, C.E., Steece-Collier, K., Collier, T.J., 2006. Failure of proteasome inhibitor administration to provide a model of Parkinson's disease in rats and monkeys. Ann Neurol 60, 264-268. 
Kaariainen, T.M., Kaenmaki, M., Forsberg, M.M., Oinas, N., Tammimaki, A., Mannisto, P.T., 2012. Unpredictable rotational responses to L-dopa in the rat model of Parkinson's disease: the role of L-dopa pharmacokinetics and striatal dopamine depletion. Basic Clin Pharmacol Toxicol 110, 162-170.

Landau, A.M., Alstrup, A.K., Audrain, H., Jakobsen, S., Simonsen, M., Moller, A., Videbech, P., Wegener, G., Gjedde, A., Doudet, D.J., 2017. Elevated dopamine D1 receptor availability in striatum of Gottingen minipigs after electroconvulsive therapy. J Cereb Blood Flow Metab, 271678X17705260.

Lee, C.S., Samii, A., Sossi, V., Ruth, T.J., Schulzer, M., Holden, J.E., Wudel, J., Pal, P.K., de la FuenteFernandez, R., Calne, D.B., Stoessl, A.J., 2000. In vivo positron emission tomographic evidence for compensatory changes in presynaptic dopaminergic nerve terminals in Parkinson's disease. Ann Neurol 47, 493-503.

Leroy, E., Boyer, R., Auburger, G., Leube, B., Ulm, G., Mezey, E., Harta, G., Brownstein, M.J., Jonnalagada, S., Chernova, T., Dehejia, A., Lavedan, C., Gasser, T., Steinbach, P.J., Wilkinson, K.D., Polymeropoulos, M.H., 1998. The ubiquitin pathway in Parkinson's disease. Nature 395, 451-452.

Li, X., Du, Y., Fan, X., Yang, D., Luo, G., Le, W., 2008. c-Jun N-terminal kinase mediates lactacystin-induced dopamine neuron degeneration. J Neuropathol Exp Neurol 67, 933-944.

Logan, J., Fowler, J.S., Volkow, N.D., Wang, G.J., Ding, Y.S., Alexoff, D.L., 1996. Distribution volume ratios without blood sampling from graphical analysis of PET data. J Cereb Blood Flow Metab 16, 834-840.

Lorenc-Koci, E., Lenda, T., Antkiewicz-Michaluk, L., Wardas, J., Domin, H., Smialowska, M., Konieczny, J., 2011. Different effects of intranigral and intrastriatal administration of the proteasome inhibitor lactacystin on typical neurochemical and histological markers of Parkinson's disease in rats. Neurochemistry International 58, 839-849.

Lowe, J., Blanchard, A., Morrell, K., Lennox, G., Reynolds, L., Billett, M., Landon, M., Mayer, R.J., 1988. Ubiquitin is a common factor in intermediate filament inclusion bodies of diverse type in man, including those of Parkinson's disease, Pick's disease, and Alzheimer's disease, as well as Rosenthal fibres in cerebellar astrocytomas, cytoplasmic bodies in muscle, and mallory bodies in alcoholic liver disease. J Pathol 155, 9-15.

Mackey, S., Jing, Y., Flores, J., Dinelle, K., Doudet, D.J., 2013. Direct intranigral administration of an ubiquitin proteasome system inhibitor in rat: behavior, positron emission tomography, immunohistochemistry. Exp Neurol 247, 19-24.

Mathur, B.N., Neely, M.D., Dyllick-Brenzinger, M., Tandon, A., Deutch, A.Y., 2007. Systemic administration of a proteasome inhibitor does not cause nigrostriatal dopamine degeneration. Brain Res 1168, 83-89.

McGeer, P.L., McGeer, E.G., 2008. Glial reactions in Parkinson's disease. Mov Disord 23, 474-483.

McGeer, P.L., Schwab, C., Parent, A., Doudet, D., 2003. Presence of reactive microglia in monkey substantia nigra years after 1-methyl-4-phenyl-1,2,3,6-tetrahydropyridine administration. Ann Neurol 54, 599-604.

McNaught, K.S., Bjorklund, L.M., Belizaire, R., Isacson, O., Jenner, P., Olanow, C.W., 2002. Proteasome inhibition causes nigral degeneration with inclusion bodies in rats. Neuroreport 13, 1437-1441.

McNaught, K.S., Jenner, P., 2001. Proteasomal function is impaired in substantia nigra in Parkinson's disease. Neurosci Lett 297, 191-194.

McNaught, K.S., Perl, D.P., Brownell, A.L., Olanow, C.W., 2004. Systemic exposure to proteasome inhibitors causes a progressive model of Parkinson's disease. Ann Neurol 56, 149-162.

Mejias, M., Yu, J., Mackey, S., Dinelle, K., Sossi, V., Doudet, D.J., 2016. Interpreting DTBZ binding data in rodent: Inherent variability and compensation. Synapse 70, 147-152. 
Mikkelsen, M., Moller, A., Jensen, L.H., Pedersen, A., Harajehi, J.B., Pakkenberg, H., 1999. MPTP-induced Parkinsonism in minipigs: A behavioral, biochemical, and histological study. Neurotoxicol Teratol 21, 169175.

Miwa, H., Kubo, T., Suzuki, A., Nishi, K., Kondo, T., 2005. Retrograde dopaminergic neuron degeneration following intrastriatal proteasome inhibition. Neurosci Lett 380, 93-98.

Niu, C., Mei, J., Pan, Q., Fu, X., 2009. Nigral degeneration with inclusion body formation and behavioral changes in rats after proteasomal inhibition. Stereotact Funct Neurosurg 87, 69-81.

Phan, J.A., Stokholm, K., Zareba-Paslawska, J., Jakobsen, S., Vang, K., Gjedde, A., Landau, A.M., RomeroRamos, M., 2017. Early synaptic dysfunction induced by alpha-synuclein in a rat model of Parkinson's disease. Sci Rep 7, 6363.

Pienaar, I.S., Harrison, I.F., Elson, J.L., Bury, A., Woll, P., Simon, A.K., Dexter, D.T., 2015. An animal model mimicking pedunculopontine nucleus cholinergic degeneration in Parkinson's disease. Brain Struct Funct 220, 479-500.

Rott, R., Szargel, R., Haskin, J., Bandopadhyay, R., Lees, A.J., Shani, V., Engelender, S., 2011. alpha-Synuclein fate is determined by USP9X-regulated monoubiquitination. Proc Natl Acad Sci U S A 108, 18666-18671.

Savolainen, M.H., Albert, K., Airavaara, M., Myohanen, T.T., 2017. Nigral injection of a proteasomal inhibitor, lactacystin, induces widespread glial cell activation and shows various phenotypes of Parkinson's disease in young and adult mouse. Exp Brain Res 235, 2189-2202.

Schapira, A.H., Cleeter, M.W., Muddle, J.R., Workman, J.M., Cooper, J.M., King, R.H., 2006. Proteasomal inhibition causes loss of nigral tyrosine hydroxylase neurons. Ann Neurol 60, 253-255.

Scherman, D., Raisman, R., Ploska, A., Agid, Y., 1988. [3H]dihydrotetrabenazine, a new in vitro monoaminergic probe for human brain. J Neurochem 50, 1131-1136.

Schneider, C.A., Rasband, W.S., Eliceiri, K.W., 2012. NIH Image to ImageJ: 25 years of image analysis. Nat Methods 9, 671-675.

Strome, E.M., Cepeda, I.L., Sossi, V., Doudet, D.J., 2006. Evaluation of the integrity of the dopamine system in a rodent model of Parkinson's disease: small animal positron emission tomography compared to behavioral assessment and autoradiography. Mol Imaging Biol 8, 292-299.

Subramaniam, M., Kern, B., Vogel, S., Klose, V., Schneider, G., Roeper, J., 2014. Selective increase of in vivo firing frequencies in DA SN neurons after proteasome inhibition in the ventral midbrain. Eur J Neurosci 40, 2898-2909.

Suzuki, M., Desmond, T.J., Albin, R.L., Frey, K.A., 2001. Vesicular neurotransmitter transporters in Huntington's disease: initial observations and comparison with traditional synaptic markers. Synapse 41, 329-336.

Tofaris, G.K., Layfield, R., Spillantini, M.G., 2001. alpha-synuclein metabolism and aggregation is linked to ubiquitin-independent degradation by the proteasome. FEBS Lett 509, 22-26.

Tong, J., Wilson, A.A., Boileau, I., Houle, S., Kish, S.J., 2008. Dopamine modulating drugs influence striatal $(+)-[11 C] D T B Z$ binding in rats: VMAT2 binding is sensitive to changes in vesicular dopamine concentration. Synapse 62, 873-876.

Vander Borght, T., Kilbourn, M., Desmond, T., Kuhl, D., Frey, K., 1995. The vesicular monoamine transporter is not regulated by dopaminergic drug treatments. Eur J Pharmacol 294, 577-583. 
Vernon, A.C., Crum, W.R., Johansson, S.M., Modo, M., 2011. Evolution of extra-nigral damage predicts behavioural deficits in a rat proteasome inhibitor model of Parkinson's disease. PLoS One 6, e17269.

Voges, D., Zwickl, P., Baumeister, W., 1999. The 26S proteasome: a molecular machine designed for controlled proteolysis. Annu Rev Biochem 68, 1015-1068.

Watanabe, H., Andersen, F., Simonsen, C.Z., Evans, S.M., Gjedde, A., Cumming, P., DaNe, X.S.G., 2001. MR-based statistical atlas of the Gottingen minipig brain. Neuroimage 14, 1089-1096.

Xie, W., Li, X., Li, C., Zhu, W., Jankovic, J., Le, W., 2010. Proteasome inhibition modeling nigral neuron degeneration in Parkinson's disease. J Neurochem 115, 188-199. 


\section{Figure Legends}

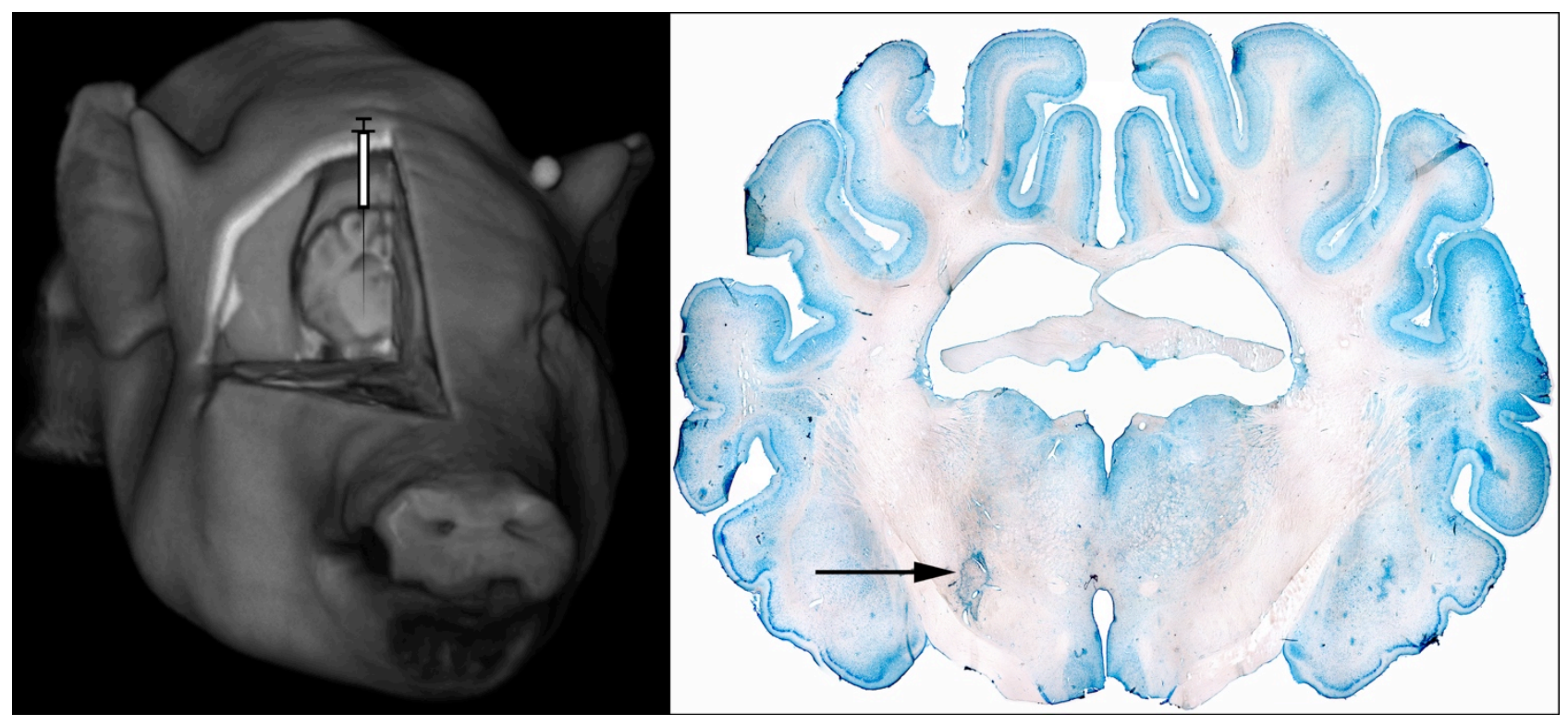

Figure 1. MRI-based stereotaxic injection in the MFB of the minipig. Left: Coronal MRI overlaid with needle visualizing the injection of lactacystin in the MFB. Three injections were performed $1 \mathrm{~mm}$ apart (anteriorposterior). Right: Nissl staining of a representative brain slice from pig\#4 with visible needle tract (arrow).

a

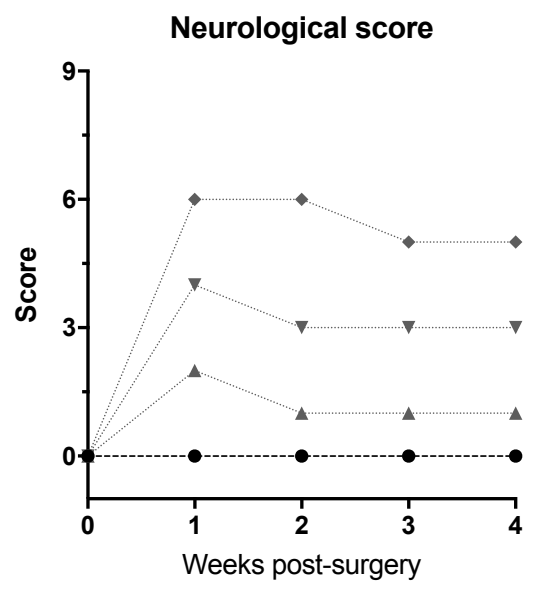

b

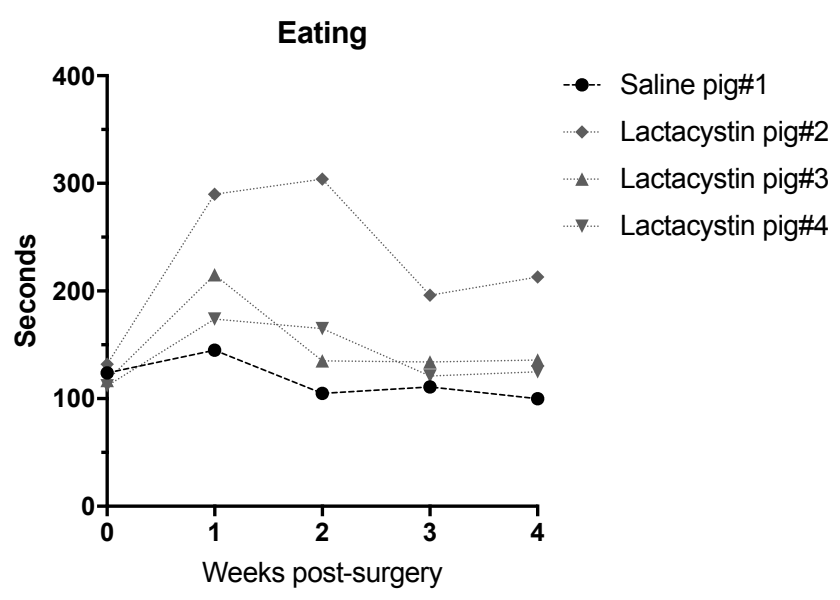

Figure 2. Motor performance and eating duration. (a) Neurological score and (b) Eating duration assessed every week. 
a

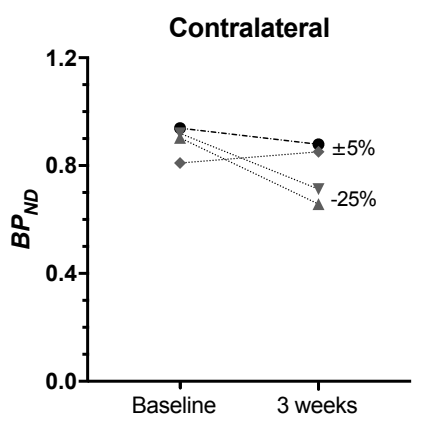

b

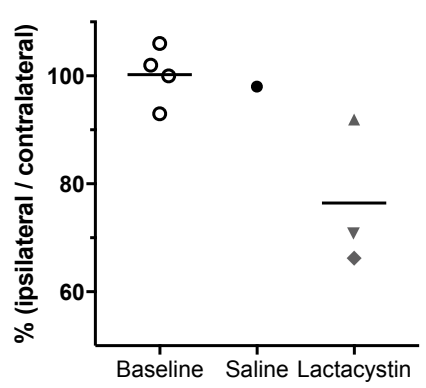

Ipsilateral

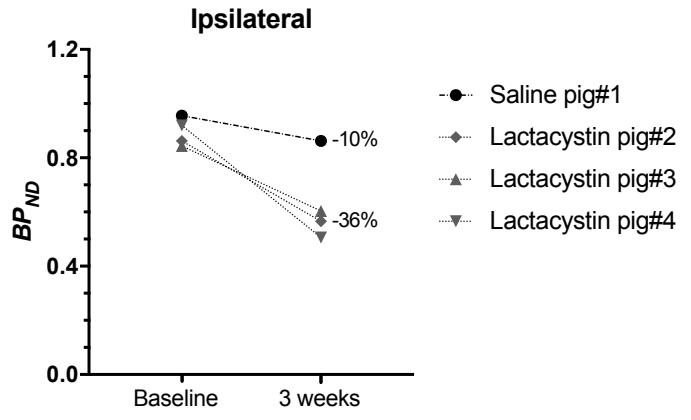

C
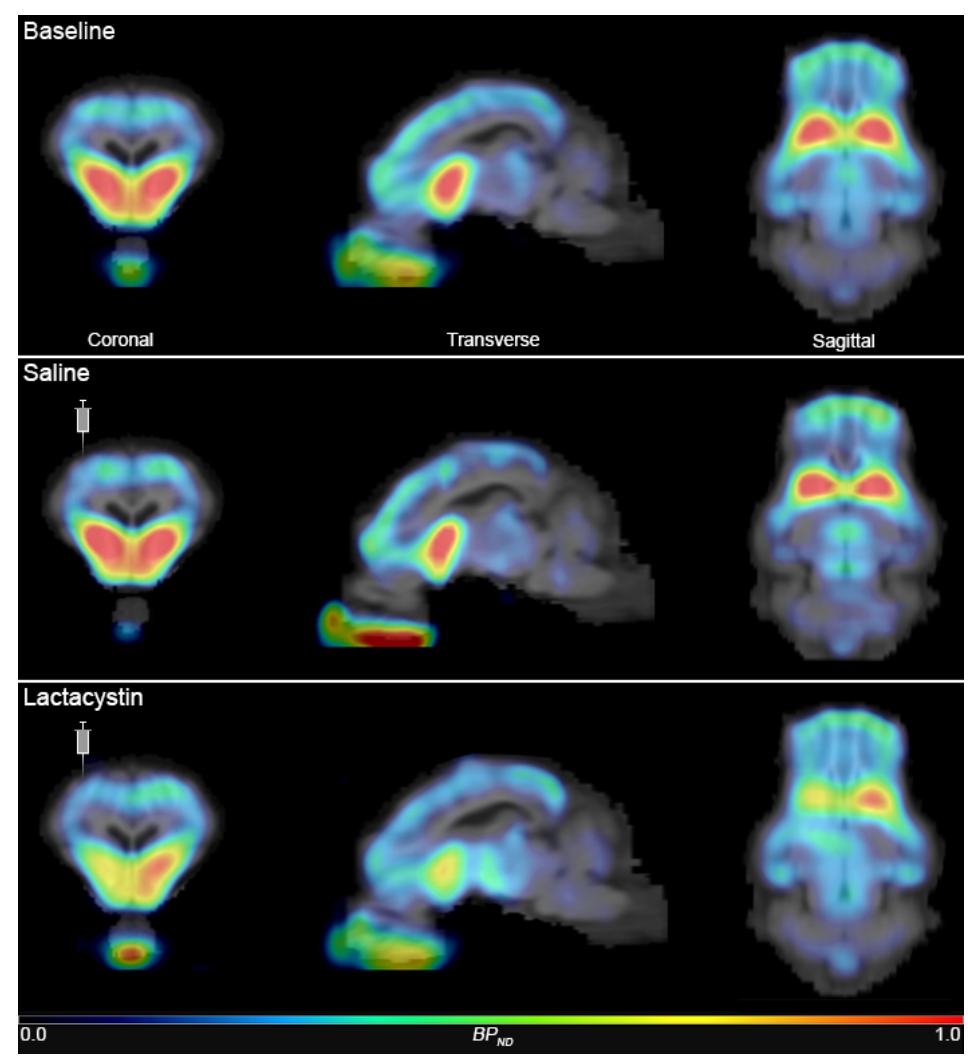

Figure 3. VMAT2 binding levels determined by in vivo $\left[{ }^{11} C\right] D T B Z$ PET. (a) $B P_{N D}$ values are presented for each minipig at baseline and 3 weeks post-surgery in both the contralateral (left graph) and ipsilateral striatum (right graph). (b) The ipsilateral relative to contralateral percentage change are shown of all animals at baseline

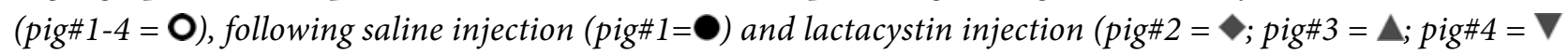
). (c) Parametric $B P_{N D}$ maps of in vivo $\left[{ }^{11} C\right] D T B Z$ PET are displayed in coronal, transverse and sagittal views. The top row represents an average of the $4 B P_{N D} P E T$ images obtained at baseline. The middle row display images of the saline-injected minipig at its 3-week scan while the bottom row shows the voxel-wise parametric maps of an average of the three lactacystin-injected minipigs 3 weeks post-injection. While the baseline and the 
saline scans show similar binding in ipsilateral and contralateral striatum (top and middle row), a visible decreased binding can be observed in the ipsilateral striatum after unilateral lactacystin injection (bottom row).
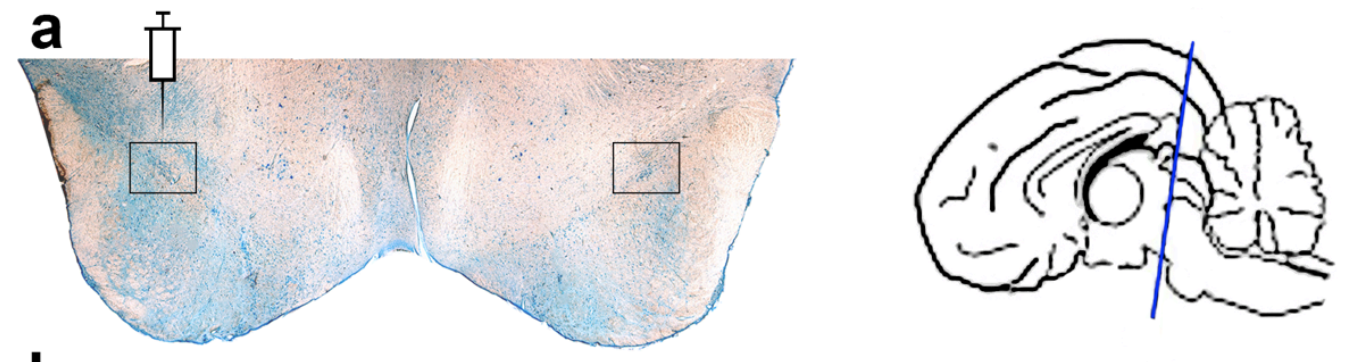

b
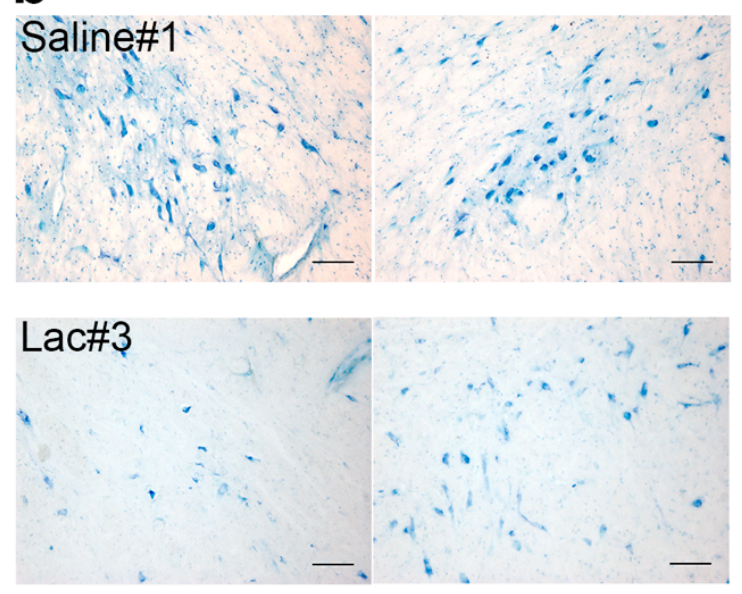
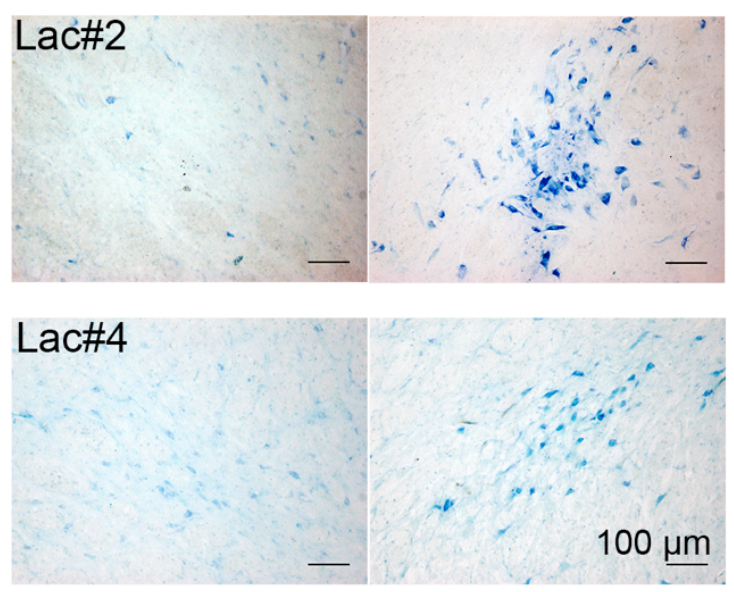

Figure 4. SN pars compacta (SNc) visualized with Nissl staining to determine cell loss. (a) Left: Saline-injected pig\#1 is displayed and rectangular boxes are placed to indicate the anatomical location of the magnified inserts below. Right: An adapted schematic drawing of the Göttingen minipig brain depicting the section plane corresponding to S20 in the histological atlas by Bjarkam et al. (2016) (http://www.cense.dk/minipig atlas/). (b) Magnified images are shown of corresponding ipsilateral and contralateral SNc sections of the salineinjected pig\#1 (top left), lactacystin-injected pig\#2 (top right), lactacystin-injected pig\#3 (bottom left) and lactacystin-injected pig\#4 (bottom right). Lac = lactacystin. Scale bars $=100 \mu \mathrm{m}$. 
a
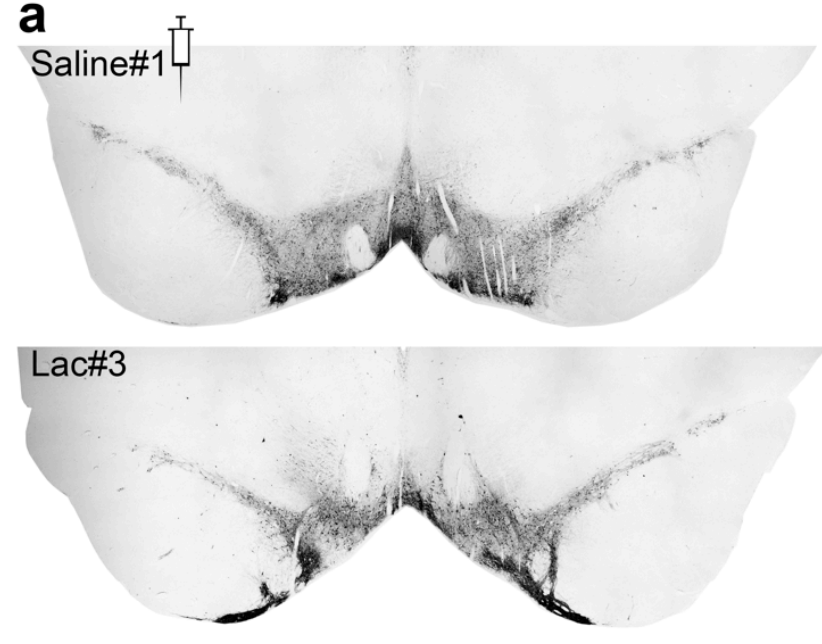

b

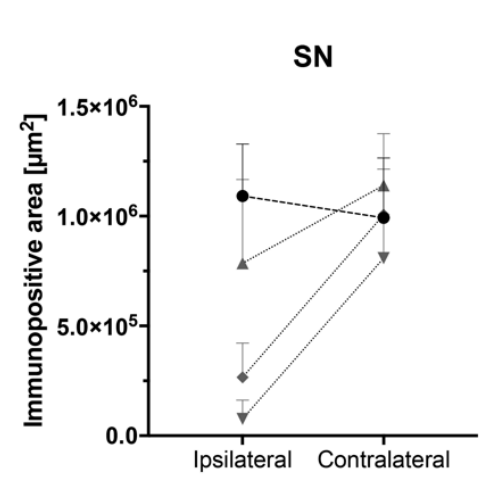

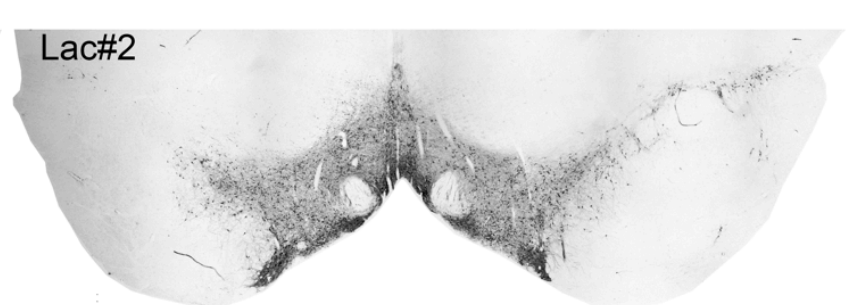

Lac\#4

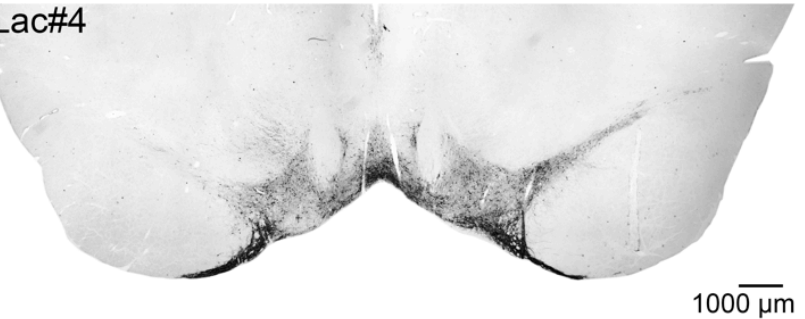

C

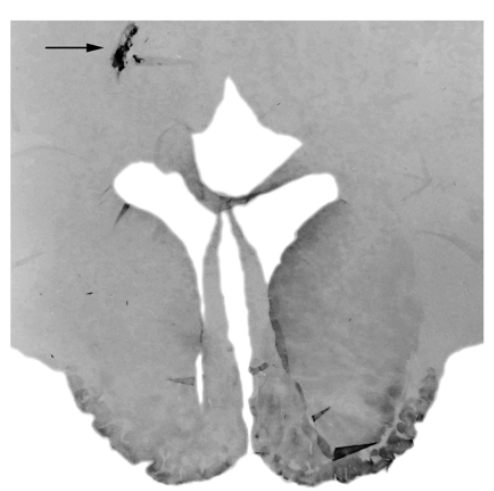

Figure 5. Representative TH immunohistochemistry sections shown for the SN, VTA and striatum. (a) Upper left: saline-injected pig\#1. Upper right: lactacystin-injected pig\#2. Bottom left: lactacystin-injected pig\#3. Bottom right: lactacystin-injected pig\#4. (b) Measurement of the immunopositive SN and VTA area in contralateral and ipsilateral side of each minipig post-surgery. Each point on the graph represents mean and standard deviation (error bar) of measurements done on 3 adjacent sections covering the SN and VTA for each minipig. (c) Representitative brain section of striatum in lactacystin-injected minipig. The reduced staining of TH fibers can be appreciated in the ipsilateral stiatum compared to the contralateral striatum. Needle injection tract is visible in the cortex (arrow). Lac = lactacystin.

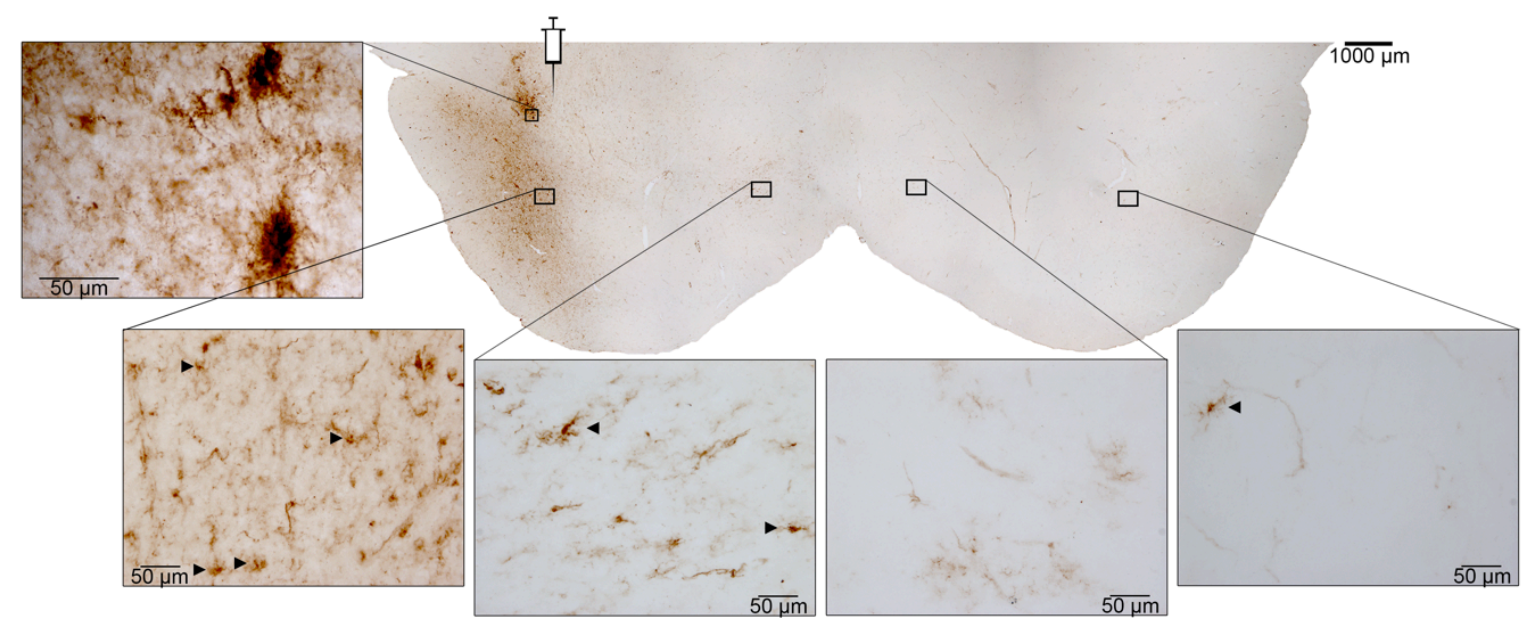

Figure 6. HLA-DR-positive microglia in SN and VTA of lactacystin-injected minipig \#2. Mild hypertrophic and phagocytic microglia were seen in the ipsilateral side, while ramified and a few hypertrophic microglia were observed contralateral to the injection site. Arrows indicate mildly hypertrophic microglia. 

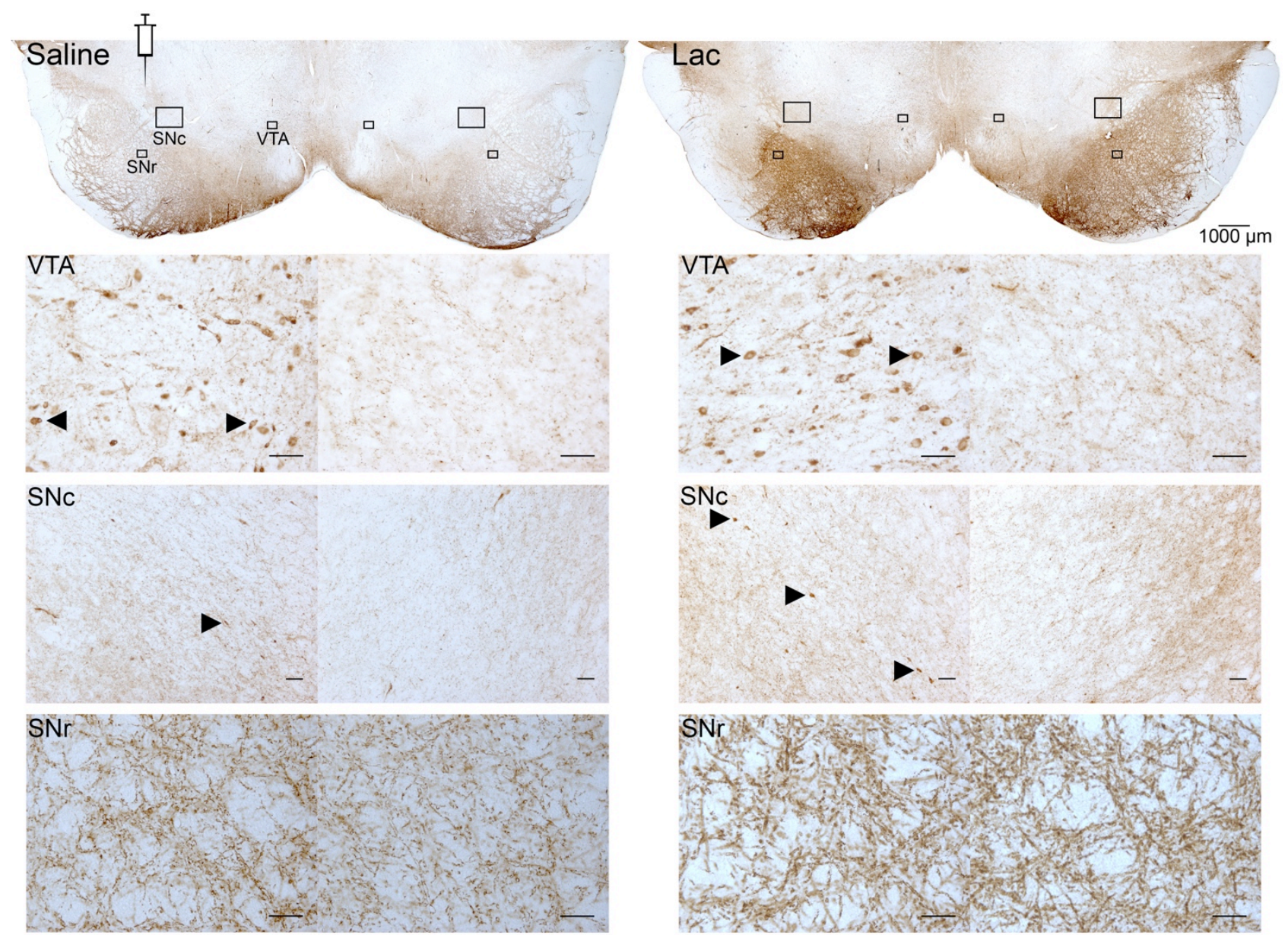

Figure 7. $\alpha$-syn histology in $S N$ pars compacta $(S N c), S N$ pars reticulata $(S N r)$ and ventral tegmental area (VTA). To the left, $\alpha$-syn staining is shown in the saline-injected minipig\# 1, and to the right, in a lactacystin (Lac)-injected minipig \#2. Neurons and synapses (puncta) in the VTA expressing $\alpha$-synuclein are visible in the ipsilateral side in Lac-injected minipig. Only puncta and no neurons were visible in non-injected side in the $V T A$. In the SNc of both saline-and lactacystin-injected pigs, small puncta were visible on both ipsi-and contralateral sides with a few neurons stained ipsilaterally in the lactacystin-injected animal. $\alpha$-positive granules were observed in SNr and no differences were seen between the ipsi-and contralateral side in either brains. Large rectangular boxes show the magnified SNc images while the small rectangular boxes show the magnified VTA and SNr inserts. Arrows mark stained neurons. Scale bars in inserts: $50 \mu \mathrm{m}$. 